\title{
Lycium Barbarum Polysaccharides Regulate the Gut Microbiota To Modulate Metabolites in High Fat Diet-Induced Obese Rats
}

\section{Yanna Fan}

Ningxia Medical University

Lu Yan

Ningxia Medical University

Mengyao Li

Ningxia Medical University

Zhiyu Pu

Ningxia Medical University

Yannan Zhang ( $\nabla$ yannan1220@outlook.com )

Ningxia Medical University

Jianjun Yang

Ningxia Medical University

\section{Research Article}

Keywords: Obesity, Metabolite, Gut microbiome, Lycium Barbarum Polysaccharides

Posted Date: December 10th, 2021

DOI: https://doi.org/10.21203/rs.3.rs-1118978/v1

License: (9) (i) This work is licensed under a Creative Commons Attribution 4.0 International License. Read Full License 


\section{Abstract}

Previous studies had indicated that the gut microbiota was a main internal factor leading to obesity through energy storage and metabolic disorders. Lycium Barbarum Polysaccharides (LBP) have been discovered with a more protective effect on intestinal flora. But it is unclear whether LBP could regulate the gut microbiota to modulate metabolites, finally relieving obesity. A related study of high-throughput 16S rRNA sequencing and serum metabolomics profiling in LBP intervention on high fat diet-induced obese rats then explored the beneficial effects of LBP and the underlying mechanism. LBP affected lipid parameters such as total cholesterol, Triglyceride, and High-density lipoprotein. The gut microbiota result detected 16 types of the phylum of bacteria in total, while four of them (Bacteroidetes, Firmicutes, Proteobacteria, Deferribacteres) were significantly different. LBP upregulated the level of Firmicutes of obese rats. LBP might associate with the gut microbiota that participates in the membrane transport and metabolism of amino acid, carbohydrate, energy, and lipid. The serum metabolomics profiling of high-fat diet-induced obesity rats found over 30 differential metabolites between model and intervention groups. Primary metabolites include cortisol, glycohyocholic acid, homo-L-arginine, ursodeoxycholic acid, isoursodeoxycholic acid, glycoursocholic acid, 4-ethylphenylsulfate, deoxycholic acid, 7-hydroxy-3oxocholanoic acid isomers, gly-phe, pipecolic acid, proline betaine, and pyrocatechol sulfate. Pathway analysis in serum found four disorder pathways: glycerophospholipid metabolism, glycine-serinethreonine metabolism, biosynthesis of unsaturated fatty acids, and linoleic acid metabolism. The studies revealed that LBP treatment increased the diversity of fecal microorganisms and reduced metabolic disorders in obese rats. LBP ameliorated metabolic disorders and rebalanced the gut microbiome.

\section{Introduction}

As an epidemic disease, obesity is generally caused by fat accumulation and is characterized by increasing an unproportionate weight and height $[1,2]$. Obesity has shown a rising trend globally, and $13.1 \%$ of adults are obese[3]. Obesity is usually comorbid with cardiovascular, metabolic, and psychosocial diseases. These comorbidities are leading to increased disease burden, morbidity, and mortality[4, 5].

Previous studies indicated that the intestinal microbiota had been linked to obesity, and a meta-analysis suggests the promotion of probiotics in weight loss in adults[6]. Some data showed that the microbiota might connect with host metabolism through its production of metabolites; for example, the abundance of B.thetaiotaomicron was decreased in obese rats and the weight-loss intervention[7, 8]. Metabolites derived from intestinal flora, such as 2-hydroxyisobutyrate and xanthine, were linked to obesity[9]. The typical obese metabolites were lost after bariatric surgery-induced weight loss $[9,10]$. Therefore, researches on gut microbiota and metabolism could play an important role in therapeutic and preventive interventions[10-13].

Lycium barbarum polysaccharides (LBP) are biologically active components extracted from Goji berries and have been extensively explored for their health effects[14]. Evidence shows that LBP can lower blood 
lipid levels in a hyperlipidemic animal model[15]. Another study elucidated that LBP could improve the amount of intestinal microbiota[16, 17]. However, whether LBP regulates the disorder of bacteria then adjusted metabolites of high-fat-diet (HFD), its mechanism is still unclear, and its anti-obesogenic effect was not reported[18].

This study used high-throughput 16S rRNA sequencing and un-target metabolomics in LBP intervention on high fat diet-induced obese rats to examine the gut bacteria and metabolites. It aimed to clarify the changing of gut microbiota and metabolites, explore the beneficial effects of LBP and the underlying mechanism of the gut microbiota modulating metabolites. Consequently, we provided important information for the scientific basis of obesity prevention. Gut microbiota and metabolite composition of rat serums were investigated by $16 \mathrm{~S}$ rRNA sequencing and Ultra Performance Liquid ChromatographyHigh Resolution Mass Spectrometry (UPLC-HRMS), respectively. It aimed to identify the feces of microbiota and revealed the relationship of serum metabonomic between groups control, model, and different LBP doses intervention on obese rats.

\section{Materials And Methods}

\subsection{LBP}

LBP was obtained from Shanghai Yuanye Biotechnology Co., Ltd (No. B20460, China). LBP was dissolved in pure water in a solution of $10 \mathrm{mg} / \mathrm{ml}, 30 \mathrm{mg} / \mathrm{ml}$, and $90 \mathrm{mg} / \mathrm{ml}$. The content in the LBP product was analyzed by the phenol-sulfuric acid method[19].

\subsection{Animals breeding and maintenance diets}

Male Sprague Dawley rats of clean grade (8-weeks old; body weight $200.0 \pm 2.0 \mathrm{~g}$ ) were supplied with certificate no. SCXK (Ning) 2020-0001 from the Laboratory Animal Center of Ningxia Medical University (Ningxia, China). All rats were fed in the laboratory animal center of Ningxia Medical University and kept a 12-h-dark/12-h-light cycle relative humidity $(60 \%-65 \%)$ at $25^{\circ} \mathrm{C}$. We supplied sterile water and food in this whole experiment. All procedures were getting permission from the Ethics Committee of Ningxia Medical University.

To create the HFD induced obesity model for 12 weeks, fifty-seven rats with body weights were separated into two groups (control $n=11$, modeling $n=46$ ) randomly based on their diet (HFD, High Fat Diets, $60 \%$ energy from fat; MD12033, medicience. Ltd, Jiangsu, China) and control (10\% energy from fat; 1010009 , Xietong, Jiangsu, China). Obesity was defined as $10 \%$ more weight than the standard diet group at 12 weeks[20]. The experimental group received LBP for another 12 weeks at different doses, low $(10 \mathrm{mg} / \mathrm{kg}$ bw), medium ( $30 \mathrm{mg} / \mathrm{kg} \mathrm{bw}$ ), and high ( $90 \mathrm{mg} / \mathrm{kg} \mathrm{bw})$, separately. In comparison, the control and the model group were given the same volume of physiological saline via intragastric administration. Bodyweight was determined weekly during the whole experiment.

Calculated Lee index[21, 22]: Lee index $=$ Bodyweight $(\mathrm{g})^{1 / 3} \times 1000 /$ body length $(\mathrm{cm})$. 


\subsection{Sample Collection}

The rats were weighed, measured body length under anesthesia, and sacrificed. Blood was collected from the aorta ventralis after 12 weeks of LBP exposure. Then blood samples were gathered after the centrifugation of $4000 \times \mathrm{g}$ for 10 minutes at $4^{\circ} \mathrm{C}$, stored at $-80^{\circ} \mathrm{C}$ for the next experiment. Fresh feces were picked into individual $5 \mathrm{ml}$ Eppendorf tubes from the intestine after the execution and snap-frozen with liquid nitrogen for decreasing its pollution by environment and stored in the ultra-low temperature refrigerator.

\subsection{Sample preparation}

\subsubsection{Sample preparation for $16 \mathrm{~S}$ rRNA}

The instruction extracted genomic DNA from the feces using a MagPure Soil DNA LQ Kit (Magen, D635602). Amplicon quality was monitored by gel electrophoresis, purified with AMPure XP beads (Agencourt), and performed another round of PCR after amplification. We were using the Qubit dsDNA assay kit to quantify the final amplicon. Illumina novaseq6000 (Illumina, San Diego, USA) was used to survey the concentration pooled purified amplicon. The 16S rRNA V3-V4 region was magnified by PCR. The primer sequences followed by: 343F (5'- TACGGRAGGCAGCAG -3') and 798R (5'- AGGGTATCTAATCCT-3') primers. The first-round PCR used an initial step at $94^{\circ} \mathrm{C}$ for $5 \mathrm{~min}$; then took $94^{\circ} \mathrm{C}$ for $30 \mathrm{~s}, 56^{\circ} \mathrm{C}$ for $30 \mathrm{~s}$, $72^{\circ} \mathrm{C}$ for $20 \mathrm{~s}$ in 26 cycles, and $72^{\circ} \mathrm{C}$ for 5 min for the final extension.

\subsubsection{Sample preparation for metabolomics}

The serum sample was thawed at $4^{\circ} \mathrm{C}$, absorbed and transferred $50 \mu \mathrm{l}$ accurately to EP tube, added $200 \mu \mathrm{l}$ methanol-acetonitrile $(1 / 1, V / V)$ mixing with internal standard, vortexed for 120 s, centrifugation at 15,000 $\mathrm{g} \times 15 \mathrm{~min}$, transfer $200 \mu \mathrm{l}$ supernatant to $1.5 \mathrm{ml}$ EP tube. Low-temperature vacuum drying in centrifugal concentrator (Thermo Scientific, USA), sealed and cryopreserved before analysis. Before LC-MS analysis, $100 \mu \mathrm{L} 50 \%$ methanol was used after dissolving.

The hydrophilic fraction of metabolite extracts were submitted to the non-targeted metabolomics analysis platform by Ultimate TM 3000 ultra-high-performance liquid chromatography coupled to Q Exactive $^{T M}$ quadrupole-Orbitrap high-resolution mass spectrometer system (UPLC/HRMS) (Thermo Scientific, San Jose, USA) scanned at 50-1000 m/z mass range with ESI in positive and negative modes separately. The metabolite profile with reverse-phase chromatographic separation mode with positiveand negative-ion detection. Metabolites were apart by an Acquity ${ }^{\mathrm{TM}}{ }^{\mathrm{HSS}} \mathrm{C} 18$ column (Waters Corporation, Milford, USA, $1.8 \mu \mathrm{m}, 2.1 \times 100 \mathrm{~mm}$ ). The gradient followed by: $2 \%$ organic phase $0 \mathrm{~min}$ ramped to $100 \%$ in $10 \mathrm{mins}$, column washing and equilibrating for another $5 \mathrm{~min}$. The flow rate, injection volume, and column temperature were $0.4 \mathrm{ml} / \mathrm{min}, 5 \mu \mathrm{l}$, and $50^{\circ} \mathrm{C}$, respectively.

Quality control (QC) samples assessed the UPLC/HRMS system's reproducibility and reliability.

\subsection{Data analysis and results interpretation}


For bodyweight, length, and all biochemical indexes, the data were expressed as the mean \pm SD and used Student's t-test. A significant difference was considered $p<0.05$.

The data were graphically plotted using GraphPad Prism 7.

\subsubsection{6s rRNA assessment}

Sequences and alpha diversity (Chao1 and Shannon indices) analysis were used Quantitative Insights into Microbial Ecology (QIIME 1.8.0 version). We limited operational taxonomic units (OTUs) by the results of clustering into sequence tags. Sequences over $97 \%$ similarity were defined to the same OTUs to species annotation and taxonomic analysis on the SILVA database. Beta diversity and Principal coordinate analysis (PCoA) were calculated from Bray Curtis distance matrices to show differences between the gut microbiota composition and structure. The unweighted pair-group method with arithmetic mean (UPGMA) was used to measure the hierarchical clustering of samples. We used PICRUSt software to predict functional 16r RNA profiles and produce KEGG pathways[23].

A non-parametric test (Mann-Whitney $\mathrm{U}$ test and Kruskal-Wallis) was analyzed gut microbiota by SPSS software (IBM SPSS 21.0; SPSS Inc., Chicago, IL, USA).

The R package (http://microbiome.github.com/ microbiome) calculated Spearman's correlation coefficient $r$ and examined correlations between gut microbiota composition and serum metabolites $(r>0$ means the positive correlation between indicators; $r<0$ was negative).

\subsubsection{Metabolite profile analysis}

The data from the untargeted metabolomic analysis was obtained by Mass Lynx v.4.1 software (including $\mathrm{ESI}^{+}$and $\mathrm{ESI}^{-}$modes). The metabolomic data from different measurements were normalized using the support vector regression method in the MetNormalizer R package[24].

The MS/MS metabolic data (hydrophilic fraction) were processed with Compound Discoverer software (Thermo Scientific, San Jose, USA) to help accomplish content extraction.

For identifying or structural annotation of metabolites, retention time and high-resolution MS/MS spectrum similarity were strictly used to determine the structural annotation of metabolites. Used XCalibur Quan Browser information to extract the area under the curve as the quantitative information of metabolites and exported the peak area data of all labeled metabolites to Excel software for trimming and statistics. The chemical identification results were finally annotated with classification criteria proposed by MSI (Metabolomics Standardization Initiative).

The output tables of Metabolites were defined for SIMCA 14.1 software (Sartorius AG Umetrics, Goettingen, Germany). We conducted principal component analysis (PCA), orthogonal projections to latent structures discriminant analysis (OPLS-DA), and coordinates analysis was performed with $R$ package[25]. To avoid chemical noise, all models adopted the Pareto transformation. Potential 
metabolites were selected based on variable importance (VIP) value (VIP > 1.5) combined with the t-test $p$-value $(\mathrm{p}<0.05)$ in the OPLS-DA model. We used MetaboAnalyst 4.0 (http://www.metaboanalyst. ca) to visualize the metabolites differences between two groups by generating the heatmap.

Venn diagram showed differential metabolites in intervention groups and model group[26].

\section{Results}

\subsection{The content of LBP}

The content of LBP was measured to be $85.74 \%$. Drew a standard graph by the concentration of glucose as $\mathrm{x}$-axis and absorbance on $\mathrm{y}$-axis, the regression equation was $\mathrm{y}=7.8596 \mathrm{x}-0.0012, \mathrm{R}^{2}=0.9988$.

\subsection{Biochemical indexes}

Fifty rats were fit for the subsequent experiment treatment after developing the model successfully. The bodyweight of the intervention rats changed smoothly contrasted with the control rats. Treatment with LBP for 12 weeks decreased the trend of obesity, especially in the medium group. The weight of rats changed significantly. Lee index changed obviously compared intervention groups to control and the model group. Lee index in high LBP group lower than the model and low LBP group. It also significantly decreased with LBP dose (Figure 1. a-c).

The results of the biochemical analysis were shown in Table 1. Medium and high LBP intervention groups had descended in TC, TG, ALT, AST, CREA, UA, and LDL levels, increasing HDL simultaneously, compared with the control and model group. However, the effect was not noticeable $(p>0.05)$. The medium group had a different trend for biochemical indexes (Table 1). 
Table 1

Characteristics of serum with LBP for control and intervention groups

\begin{tabular}{|c|c|c|c|c|c|c|c|}
\hline Parameter & $\begin{array}{l}\text { Control } \\
n=11\end{array}$ & $\begin{array}{l}\text { Model } \\
\mathrm{n}=9\end{array}$ & $\begin{array}{l}\text { low LBP } \\
\mathrm{n}=10\end{array}$ & $\begin{array}{l}\text { med LBP } \\
\mathrm{n}=10\end{array}$ & $\begin{array}{l}\text { high LBP } \\
\mathrm{n}=10\end{array}$ & $\mathbf{F}$ & $p$ \\
\hline TC & $\begin{array}{l}1.56 \pm \\
0.58\end{array}$ & $\begin{array}{l}1.65 \pm \\
0.64\end{array}$ & $1.50 \pm 0.44$ & $\begin{array}{l}1.38 \pm \\
0.49\end{array}$ & $\begin{array}{l}1.47 \pm \\
0.56\end{array}$ & 2.055 & 0.072 \\
\hline TG & $\begin{array}{l}2.54 \pm \\
0.63\end{array}$ & $\begin{array}{l}2.54 \pm \\
0.58\end{array}$ & $2.40 \pm 0.49$ & $\begin{array}{l}2.12 \pm \\
0.37\end{array}$ & $\begin{array}{l}2.07 \pm \\
0.48^{\star}\end{array}$ & 9.829 & 0.000 \\
\hline ALT & $\begin{array}{l}51.72 \pm \\
18.12\end{array}$ & $\begin{array}{l}53.35 \pm \\
15.89\end{array}$ & $54.42 \pm 11.16$ & $\begin{array}{l}47.43 \pm \\
13.80\end{array}$ & $\begin{array}{l}50.48 \pm \\
7.67\end{array}$ & 0.255 & 0.904 \\
\hline AST & $\begin{array}{l}188.33 \pm \\
43.27\end{array}$ & $\begin{array}{l}203.94 \pm \\
44.63\end{array}$ & $\begin{array}{l}191.74 \pm \\
57.08\end{array}$ & $\begin{array}{l}189.23 \pm \\
26.77\end{array}$ & $\begin{array}{l}195.40 \pm \\
40.96\end{array}$ & 0.161 & 0.957 \\
\hline CREA & $\begin{array}{l}41.24 \pm \\
3.58\end{array}$ & $\begin{array}{l}42.30 \pm \\
3.97\end{array}$ & $40.37 \pm 5.15$ & $\begin{array}{l}41.60 \pm \\
3.05\end{array}$ & $\begin{array}{l}41.90 \pm \\
3.29\end{array}$ & 1.079 & 0.388 \\
\hline UA & $\begin{array}{l}86.96 \pm \\
22.74\end{array}$ & $\begin{array}{l}89.98 \pm \\
24.36\end{array}$ & $\begin{array}{l}97.75 \pm \\
13.70\end{array}$ & $\begin{array}{l}82.50 \pm \\
15.40\end{array}$ & $\begin{array}{l}82.68 \pm \\
16.18\end{array}$ & 1.884 & 0.100 \\
\hline HDL & $\begin{array}{l}1.18 \pm \\
0.29\end{array}$ & $\begin{array}{l}1.12 \pm \\
0.19\end{array}$ & $\begin{array}{l}1.36 \pm \\
0.28 \mathbf{4}\end{array}$ & $\begin{array}{l}1.23 \pm \\
0.15\end{array}$ & $\begin{array}{l}1.27 \pm \\
0.20\end{array}$ & 1.373 & 0.240 \\
\hline LDL & $\begin{array}{l}0.37 \pm \\
0.12\end{array}$ & $\begin{array}{l}0.36 \pm \\
0.09\end{array}$ & $0.37 \pm 0.07$ & $\begin{array}{l}0.35 \pm \\
0.10\end{array}$ & $\begin{array}{l}0.33 \pm \\
0.08\end{array}$ & 0.221 & 0.968 \\
\hline \multicolumn{8}{|c|}{ * LBP vs Control; $\mathbf{\Delta}$ LBP vs Model, $p<0.05$} \\
\hline \multicolumn{8}{|c|}{$\begin{array}{l}\text { LBP, Lycium Barbarum Polysaccharides; TC, total cholesterol; TG, Triglyceride; ALT, Alanine } \\
\text { aminotransferase; AST Glutamic oxalacetic transaminase; CREA, Creatinine; UA, Uric acid; HDL, High- } \\
\text { density lipoprotein; LDL, Low-density lipoprotein. }\end{array}$} \\
\hline \multicolumn{8}{|c|}{ Data were expressed in mean (SD). Estimation of $p$-value by using ANOVA test* } \\
\hline
\end{tabular}

There was evidence that the gut microbiota may be conducive to relieving or treating obesity[27]. We explored was the role of LBP related to obese gut microbiota for obesity; the study used 16S rRNA (V3-V4 region). It analyzed the fecal flora of obese rats after 12 weeks of treatment. The species diversity of the samples was evaluated at the OTU level. A total of 4679 OTUs for 40 samples were generated (Figure 2. a).

The Firmicutes and Bacteroidetes ratio in the gut were closely related to obesity[28]. More than sixteen phylum of bacteria were detected, and four (Bacteroidetes, Firmicutes, Proteobacteria, Deferribacteres) were quite different between the obesity and control groups. Specifically, Bacteroidetes was the predominant phylum in all studied groups, over $60 \%$ of species abundance, but it was not significant in all groups (Figure 2. b-c). And Firmicutes, the following intestinal flora, was significantly lower in the 
obesity group than the control group. The relative abundances of Proteobacteria and Deferribacteres were higher in the obesity group than in the control group. However, there were no significant differences between the obesity group and the control. The Firmicutes/Bacteroidetes ratio was decreased on obese rats, which changed significantly on low and high LBP groups versus control (Figure 2. d).

We measured the a diversity using four indicators: Observed species, Chao 1, Shannon index, and Simpson index. The higher values of the first three indicators, the lower the Simpson value represented, the higher the richness. The plots showed Observed species, Chao 1, Shannon index statistical significance respectively $(p=0.000,0.001,0.000)$, but 0.059 for Simpson index. Then, we found the Shannon index of obese rats was lower than that of the control and high LBP groups, while low and medium LBP groups did not have efficacy almost (Figure 2. e-g).

Beta diversity was calculated to examine and plot the distributions of the 40 samples of obesity with control groups, PC1 (22.56\%) and PC2 (10.05\%). In comparison, the red points (control) were distributed in a different quadrant of the figure, and other points of obesity circulated in the bottom quadrants of the graph far away from the red points. It is suggested that there were differences in clustering patterns between them, which points of the model group and the different obesity groups overlapped. So, further explored the obesity group by dividing them using OPLS-DA for two groups. Finally, the samples of LBP intervention groups showed overall differences (Figure 2. h).

The correlations between bacterial abundance and 16S rRNA predictive function showed that more than 40 predicted functions were modified, especially amino acid metabolism, carbohydrate metabolism, energy metabolism, lipid metabolism, and membrane transport in five groups (Figure 2. i). Thus, LBP altered five potential gut microbiota metabolic functions mainly involving an amino acid, carbohydrate, energy, lipid, and membrane metabolism.

\subsection{Metabolism analysis}

The presentative method of UPLC-HRMS detected and collected the metabolites information of serum in positive and negative ions. The metabolites in serum samples were identified based on MS/MS data, and 375 metabolites were annotated.

Firstly, the data of serum samples from the five groups were analyzed using PCA and OPLS-DA. The score plots showed significant clustering in serum samples of the control, model, and different intervened groups. The metabolic phenotypes model parameter was $R^{2} X=0.611$ for unsupervised PCA score plots between the five groups (Figure 3. a). OPLS-DA model scoring chart maximized the difference between the five groups of metabolomics data, $R^{2} Y=0.541, Q^{2}=0.349$ (Figure 3. b). The same trend existed in medium and high LBP versus model modeling, respectively (Appendix-1, 2).

The score scatter plots for the PCA and OPLS-DA models showed inner-differentiation of metabolomics data between model and control group, $R^{2} Y=0.991, Q^{2}=0.934$. It noted that specific significant biochemical changes occurred following HFD induced obesity in rats. 
Compared with the mass spectrometry data and their changing trends in 3 intervention groups versus the model group. Score plot of OPLS-DA modeling indicated inner-group differentiation of metabolomics data between model and low, $R^{2} Y=0.648, Q^{2}=0.204$. (Figure 3.c). The parameters of two groups for $R^{2}$ and $Q 2$ and the permutation results to test the robustness of OPLS-DA modeling $\left(Q^{2}=-0.223\right)$, and the slope was positive, which illustrated the model validity of the low LBP to model (Figure 3.d).

The multi-group analysis, including medium LBP versus model and high LBP versus model, evaluated three groups' metabolomic changes. Based on these data, the OPLS-DA model displayed a clear separation between medium LBP, high LBP, and model groups (Figure 3. e-f). These results suggested that the LBP intervention condition of obesity significantly affected the serum metabolic profile in rats.

Finally, the differences in perturbed pathways between obesity and the LBP intervention group were analyzed by comparing the different metabolites based on the impact value greater than 0.1. Pathway analysis found four disorder pathways according to the screened differential metabolites:

glycerophospholipid metabolism, glycine, serine and threonine metabolism, biosynthesis of unsaturated fatty acids, and linoleic acid metabolism. These pathways denoted their potential as the targeted pathways of LBP against obesity. (Figure3. $\mathrm{g}-\mathrm{i}$ ).

The selection criteria (VIP > 1.5, $p<0.05$ ) identified 39, 32, and 39 different metabolites in low, medium, and high LBP groups versus the model, respectively. There were 15 metabolites detected only in high LBP group compared model, including PE (22:5n6/0:0), PE (20:3/0:0), serotonin, PE (P-18:0/0:0), PE (18:1/0:0), PE (0:0/18:1), PE (18:1/0:0), PE (0:0/22:5n3), PE (20:4/0:0), PE (0:0/18:2), PE (18:2/0:0), phenol sulphate and 3-methyluridine (Figure 4.a-b). Other metabolites were also detected in low or medium groups compared to the model. Most of the increased metabolites in the high group were lysophospholipid. The name, class, formula, $\mathrm{m} / \mathrm{z}$, and peak area in the serum metabolites are shown in appendix-3.

\subsection{Correlation between the gut microbiota and the metabolome}

To comprehensively analyze the correlation between the gut microbiota composition and the host metabolome by calculating Spearman's correlation coefficient after 12 weeks' treatment with LBP in rats (Figure 5. a-c).

Muribacter, Ruminiclostridium_1, KCM-B-112, Morganella, Ralstonia were significantly positively associated with Gly-Phe, L-Proline, Docosapentaenoic acid (FFA (22:5n6) ), Adrenic acid (FFA (22:4) ) compared med LBP with the model for 22 genera especially. KCM-B-112, Thiobacillus, Campylobacter, Morganella, Catellicoccus, Muribacter, Providencia and Mycobacterium were significantly negatively associated with PC (0:0/22:4), PE (22:5n3/0:0), PC (22:5n3/0:0), PC (20:5/0:0), PE (20:4/0:0) compared med LBP with model for 24 genera. 
Angelakisella, Kineosporia, Methylocaldum, Gemmatimonas, Helicobacter, Massilia, Thiobacillus, Streptomyces, Caenimonas, KCM-B-112, Catellicoccus, Klebsiella, Flavobacterium, Ruminiclostridium_1, and Faecalibaculum were significantly positively associated with Acetylcholine, Phe-Trp-Gly, 5Acetylamino-6-formylamino-3-methyluracil, PC (22:4/0:0), PC (18:0/0:0), PC (22:4/0:0), Succinic acid semialdehyde, 10Z-Heptadecenoic acid (FFA (17:1) ) and Phenol-sulphate compared med LBP with the model for 42 genera. These correlations were different with high LBP treatment.

Half of the 46 genera had a significant negative or positive correlation with metabolome compared low LBP with the model. Bacteroides, Klebsiella, Parabacteroides, Ruminococcaceae_UCG-005,

Ruminiclostridium_1, Ruminococcaceae_UCG-009, Ruminococcus_2, Anaerovorax, and Azospirillum_sp._47_25 were significantly positively associated with deoxycholic acid glycine conjugate, 7-hydroxy-3-oxocholanoic acid isomers, PC (22:5n-6/0:0), PC (22:6/0:0), PC (0:0/22:4), 4-ethylphenyl sulfate, n-acetyl-I-phenylalanine, deoxycholic acid glycine conjugate. Parabacteroides, Klebsiella, Ruminiclostridium_1, Erysipelotrichaceae_UCG-003, Terrimonas, Lysobacter, Ruminococcaceae_UCG-009, Ruminococcaceae_UCG-014, Sphingomonas, Angelakisella, Mycobacterium, Lechevalieria, Altererythrobacter, Ileibacterium, Pseudarthrobacter, Enterococcus and Morganella were significantly negatively correlated with n-acetyltryptophan, $n$-acetyl-I-phenylalanine, 4-ethylphenyl sulfate, PC (22:5n6/0:0), PC (22:6/0:0), PC (0:0/22:6), PI (18:0/0:0), PE (0:0/22:5n6), PE (22:5n3/0:0), PE (0:0/22:5n6), docosapentaenoic acid (FFA (22:5n6) ), and cholesterol sulfate.

Analyze the correlation with the predicted function based on gut microbiota and the serum metabolome by calculating Spearman's correlation coefficient after 12 weeks' treatment in rats with LBP. After LBP intervention, the potential predicted function of biosynthesis of 12-, 14- and 16-membered macrolides, aminobenzoate degradation, MAPK signaling pathway-yeast, lysosome, glycosphingolipid biosynthesisglobo series, adipocytokine signaling pathway, isoflavonoid biosynthesis, pentose and glucuronate interconversions, protein digestion and absorption increased; bile secretion, glycosaminoglycan biosynthesis-chondroitin sulfate, cytochrome P450, glycosylphosphatidylinositol (GPI) -anchor biosynthesis and Renin-angiotensin system decreased. These relations suggested that gut microbiota and host metabolites profile affect each other.

\section{Discussion}

\subsection{LBP improved biochemical index in obese rats}

At present, biological activities of LBP had been previously observed that used to treat hypertension and atherosclerosis in Chinese traditional medicine that could be improved on animal and cellular models[29-31]. In this study, we provided a more integrated understanding of obesity and the role of LBP in regulating intestinal microbial to modulate metabolic processes. This experiment evaluated the effect of LBP-regulated gut microbiota to elucidate its effective mechanism connected to metabolomics. 
The obesity model was established successfully by feeding HFD for 12 weeks, as evidenced by findings consistent with previous reports[32-34]. LBP softly reversed the biochemical parameters, including TC, TG, ALT, AST, CREA, UA, HDL, LDL. It suggested that LBP may affect lipid function in our experimental and have a role to influence the obese rats and could be used for further experiments.

\subsection{LBP improved gut microbiome diversity and metabolite disorders in obese rats}

LBP intervention for obese rats altered the diversity and comprised the flora because of the a and beta diversity analysis[16]. The fecal microbiota analysis showed that LBP ameliorated dysbacteriosis by obesity. The a-diversity was significantly reduced in the model group versus the control group in this study. However, the a-diversity was restored like control in rats with LBP treatment. Bacteroidetes was the predominant phylum over $60 \%$ of all species abundance in all studied groups. Proteobacteria and Deferribacteres were decreased after LBP treatment, although still higher in each obesity group. Proteobacteria helped to reduce producing lipopolysaccharide[35]. Specifically, Firmicutes in the obese group were significantly lower than those in the control group, and it increased after intervention, which may suggest the LBP effect upregulated its level. Proteobacteria and Firmicutes played a crucial role in regulating obesity for obese rats[36,37]. LBP might be related to latent metabolic functions, such as the amino acid, carbohydrate, energy, lipid metabolism, and membrane transport of gut microbiota.

For the levels of metabolites, the intervention group displayed significant changes, including fatty acid, bile acid, and some of the amino acids, and it suggested dysfunctional glycerophospholipid metabolism, glycine, serine, and threonine metabolism, biosynthesis of unsaturated fatty acids, linoleic acid metabolism. Glycerophospholipid metabolism was related to obesity, and another metabolic syndrome was reported in a study about cohort[38]. We found various cephalin associated with obesity, like PE (22:5n3/0:0), PE (22:5n3/0:0) and lecithin, like PC (22:5n-6/0:0), PC (22:6/0:0), PC (22:4/0:0). And they only increased at the high LBP group compared with the model, but low and med LBP groups were decreased relatively. LBP may participate in the long-chain polyunsaturated fatty acids metabolic pathway or have an association with n-3 PUFA. We found the differences level of cephalin, lecithin, bile acid in serum compared to the control group, which may suggest four main pathways about lipid metabolism, all of which have a relationship like a network affected obesity progress.

The bile acid level changed in different groups, including 4-ethylphenyl sulfate, deoxycholic acid glycine conjugate, glycohyocholic acid. Early researchers had a similar report[39, 40]. And some microbial metabolites, for example, 4-ethylphenyl sulfate and 7-hydroxy-3-oxocholanoic acid isomers, also increased in intervention groups compared with the model. That suggested bile acid may have a relationship with obesity, and all different doses of LBP can affect it. The result suggested we use the targeted metabolomics techniques to focus on the bile acid metabolism next time.

\subsection{LBP connected with gut microbiome and metabolic in obese rats}


The microbial and metabolic analysis suggested microbiota were closely related to their metabolites[41]. For example, Sellimonas, Ralstonia, Sphingobium, Sulfurifustis, Sphingomonas, and Thiobacillus were positively correlated with phospholipid, like PE (22:5n3/0:0), PE (22:5n3/0:0), PC (22:5n-6/0:0), PC (22:6/0:0) and PC (22:4/0:0), but negative correlation with deoxycholic acid glycine conjugate and 4ethylphenyl sulfate of bile acid. Other microbiotas, like Morganella, Mycobacterium, Providencia, Helicobacter, Klebsiella, Catellicoccus, were opposite of the former compared medium LBP group with the model. Analyze the correlation with the predicted function based on gut microbiota and the serum metabolome by calculating Spearman's correlation coefficient after 12 weeks' treatment in rats with LBP. After LBP intervention, the potential predicted function of biosynthesis of 12-, 14- and 16-membered macrolides, aminobenzoate degradation, MAPK signaling pathway-yeast, lysosome, glycosphingolipid biosynthesis-globo series, adipocytokine signaling pathway, isoflavonoid biosynthesis, pentose and glucuronate interconversions, protein digestion and absorption increased; bile secretion, glycosaminoglycan biosynthesis-chondroitin sulfate, cytochrome P450, glycosylphosphatidylinositol (GPI)-anchor biosynthesis and renin-angiotensin system decreased. These relations may suggest that gut microbiota affect host metabolites profile.

\section{Conclusion}

Our study of LBP treatment testing showed a different dose of LBP affected lipid biochemical differently, and LBP restored microbial diversity, especially for the high LBP group, more than 20 genera positively or negatively associated with different metabolites by $16 \mathrm{~S}$ rRNA. And it also found over 30 metabolites clustered in four metabolic pathways by an untargeted metabolomics analysis platform. It was associated with glycerol phospholipid metabolism, amino acid metabolism, unsaturated fatty acid biosynthesis, and linoleic acid metabolism and was the same trend as the predictive function of intestinal flora. Different doses of LBP could affect the intestinal microflora of rats to improve the host metabolome. The current study supplied substantial evidence and a better understanding of molecular mechanisms and helped develop treatments for LBP.

\section{Declarations}

\section{Disclosure statement}

The authors declare they have no conflict of interest.

\section{Funding}

This study was supported by the Special Talents Start-up Project of Ningxia Medical University (grant No. XT2018009) and the Natural Science Foundation of Ningxia (grant No. 2020AAC03163).

\section{References}


1. Hall JE, do Carmo JM, da Silva AA, Wang Z, Hall ME: Obesity-induced hypertension: interaction of neurohumoral and renal mechanisms. Circ Res 2015, 116(6):991-1006.

2. Marques ES, Leite TH, Azeredo CM, Cunha DB, Verly Júnior E: Effective strategies for prevention, control, and treatment of obesity in primary health care setting for adolescents, adults, and elderly people: A protocol for systematic review and meta-analysis. Medicine 2018, 97(22):e10925.

3. WHO: Obesity and overweight. 2016.

4. Wolfe BM, Kvach E, Eckel RH: Treatment of Obesity: Weight Loss and Bariatric Surgery. Circulation research 2016, 118(11):1844-1855.

5. Khandelwal S: Obesity in midlife: lifestyle and dietary strategies. Climacteric: the journal of the International Menopause Society 2020, 23(2):140-147.

6. Dror T, Dickstein Y, Dubourg G, Paul M: Microbiota manipulation for weight change. Microbial pathogenesis 2017, 106:146-161.

7. Liu R, Hong J, Xu X, Feng Q, Zhang D, Gu Y, Shi J, Zhao S, Liu W, Wang X et al: Gut microbiome and serum metabolome alterations in obesity and after weight-loss intervention. Nature medicine 2017, 23(7):859-868.

8. Koh A, Bäckhed F: From Association to Causality: the Role of the Gut Microbiota and Its Functional Products on Host Metabolism. Molecular cell 2020, 78(4):584-596.

9. Calvani R, Miccheli A, Capuani G, Tomassini Miccheli A, Puccetti C, Delfini M, laconelli A, Nanni G, Mingrone G: Gut microbiome-derived metabolites characterize a peculiar obese urinary metabotype. International journal of obesity (2005) 2010, 34(6):1095-1098.

10. Vojinovic D, Radjabzadeh D, Kurilshikov A: Relationship between gut microbiota and circulating metabolites in population-based cohorts. 2019, 10(1):5813.

11. Rajagopala SV, Vashee S, Oldfield LM, Suzuki Y, Venter JC, Telenti A, Nelson KE: The Human Microbiome and Cancer. Cancer prevention research (Philadelphia, Pa) 2017, 10(4):226-234.

12. Yang L, Li L, Wu X, Cai W, Lin Q, Zhu D, Liu H: The Effect of Natural Soluble Polysaccharides on the Type 2 Diabetes through Modulating Gut Microbiota: A Review. Current medicinal chemistry 2021, 28(26):5368-5385.

13. Wang T, Yan H, Lu Y, Li X, Wang X, Shan Y, Yi Y, Liu B, Zhou Y, Lü X: Anti-obesity effect of Lactobacillus rhamnosus LS-8 and Lactobacillus crustorum MN047 on high-fat and high-fructose diet mice base on inflammatory response alleviation and gut microbiota regulation. 2020, 59(6):2709-2728.

14. Tian X, Liang T, Liu Y, Ding G, Zhang F, Ma Z: Extraction, Structural Characterization, and Biological Functions of Lycium Barbarum Polysaccharides: A Review. Biomolecules 2019, 9(9).

15. Zhu X, Hu S, Zhu L, Ding J, Zhou Y, Li G: Effects of Lycium barbarum polysaccharides on oxidative stress in hyperlipidemic mice following chronic composite psychological stress intervention. $\mathrm{Mol}$ Med Rep 2015, 11(5):3445-3450. 
16. Zhu W, Zhou S, Liu J, McLean RJC, Chu W: Prebiotic, immuno-stimulating and gut microbiotamodulating effects of Lycium barbarum polysaccharide. Biomedicine \& pharmacotherapy $=$ Biomedecine \& pharmacotherapie 2020, 121:109591.

17. Ding Y, Chen D, Yan Y, Chen G, Ran L, Mi J, Lu L, Zeng X, Cao Y: Effects of long-term consumption of polysaccharides from the fruit of Lycium barbarum on host's health. Food research international (Ottawa, Ont) 2021, 139:109913.

18. Watanabe M, Risi R: Current Evidence to Propose Different Food Supplements for Weight Loss: A Comprehensive Review. 2020, 12(9).

19. Rasouli M, Ostovar-Ravari A, Shokri-Afra H: Characterization and improvement of phenol-sulfuric acid microassay for glucose-based glycogen. European review for medical and pharmacological sciences 2014, 18(14):2020-2024.

20. Posey KA, Clegg DJ, Printz RL, Byun J, Morton GJ, Vivekanandan-Giri A, Pennathur S, Baskin DG, Heinecke JW, Woods SC et al: Hypothalamic proinflammatory lipid accumulation, inflammation, and insulin resistance in rats fed a high-fat diet. American journal of physiology Endocrinology and metabolism 2009, 296(5):E1003-1012.

21. Rabaglino MB, Moreira-Espinoza MJ, Lagares C, Garay MI, Quiroga P, Pasqualini ME, Francini F, Beltramo D: Maternal intake of alpha-lipoic acid prevents development of symptoms associated with a fructose-rich diet in the male offspring in Wistar rats. Journal of developmental origins of health and disease 2021, 12(5):758-767.

22. Nardelli TR, Ribeiro RA, Balbo SL, Vanzela EC, Carneiro EM, Boschero AC, Bonfleur ML: Taurine prevents fat deposition and ameliorates plasma lipid profile in monosodium glutamate-obese rats. Amino acids 2011, 41(4):901-908.

23. Langille MG, Zaneveld J, Caporaso JG, McDonald D, Knights D, Reyes JA, Clemente JC, Burkepile DE, Vega Thurber RL, Knight $\mathrm{R}$ et al: Predictive functional profiling of microbial communities using $16 \mathrm{~S}$ rRNA marker gene sequences. Nature biotechnology 2013, 31(9):814-821.

24. Shen X, Gong X, Cai Y, Guo Y, Tu J, Li H, Zhang T, Wang J, Xue F, Zhu Z-J: Normalization and integration of large-scale metabolomics data using support vector regression. Metabolomics 2016, 12(5).

25. Boccard J, Rutledge DN: A consensus orthogonal partial least squares discriminant analysis (OPLSDA) strategy for multiblock Omics data fusion. Analytica chimica acta 2013, 769:30-39.

26. liveros JC-V: An interactive tool for comparing lists with Venn's diagrams. https://bioinfogp.cnb.csic.es/tools/venny/index.html. (2007-2015) Venny.

27. Wang Y, Yao W, Li B, Qian S, Wei B, Gong S, Wang J, Liu M, Wei M: Nuciferine modulates the gut microbiota and prevents obesity in high-fat diet-fed rats. 2020, 52(12):1959-1975.

28. Ley RE, Bäckhed F, Turnbaugh P, Lozupone CA, Knight RD, Gordon Jl: Obesity alters gut microbial ecology. Proceedings of the National Academy of Sciences of the United States of America 2005, 102(31):11070-11075. 
29. Gao LL, Li YX, Ma JM, Guo YQ, Li L, Gao QH, Fan YN, Zhang MW, Tao XJ, Yu JQ et al: Effect of Lycium barbarum polysaccharide supplementation in non-alcoholic fatty liver disease patients: study protocol for a randomized controlled trial. Trials 2021, 22(1):566.

30. Zhang M, Li F, Pokharel S, Ma T, Wang X, Wang Y, Wang W, Lin R: Lycium barbarum polysaccharide protects against Homocysteine-induced Vascular smooth muscle cell proliferation and phenotypic transformation via PI3KJAkt pathway. Journal of molecular histology 2020, 51(6):629-637.

31. Zhou B, Xia H, Yang L, Wang S, Sun G: The effect of Lycium barbarum polysaccharide on the glucose and lipid metabolism: A systematic review and meta-analysis. Journal of the American College of Nutrition 2021:1-9.

32. Cai M, Wang H, Li JJ, Zhang YL, Xin L, Li F, Lou SJ: The signaling mechanisms of hippocampal endoplasmic reticulum stress affecting neuronal plasticity-related protein levels in high fat dietinduced obese rats and the regulation of aerobic exercise. Brain, behavior, and immunity 2016, 57:347-359.

33. Li XX, Zhao L, Chang Y, Liu BS, Xu F, Zhang C, Ji XP, Chen YG, Li CB: Ezetimibe prevents myocardial remodeling in an obese rat model by inhibiting inflammation. Acta biochimica Polonica 2018, 65(3):465-470.

34. Zhang XY, Guo CC, Yu YX, Xie L, Chang CQ: [Establishment of high-fat diet-induced obesity and insulin resistance model in rats]. Beijing da xue xue bao Yi xue ban = Journal of Peking University Health sciences 2020, 52(3):557-563.

35. Jiao X, Wang Y, Lin Y, Lang Y, Li E, Zhang X, Zhang Q, Feng Y, Meng X, Li B: Blueberry polyphenols extract as a potential prebiotic with anti-obesity effects on C57BL/ $6 \mathrm{~J}$ mice by modulating the gut microbiota. The Journal of nutritional biochemistry 2019, 64:88-100.

36. Shi Z, Zhu Y, Teng C, Yao Y, Ren G, Richel A: Anti-obesity effects of a-amylase inhibitor enrichedextract from white common beans (Phaseolus vulgaris $L$.) associated with the modulation of gut microbiota composition in high-fat diet-induced obese rats. Food \& function 2020, 11(2):1624-1634.

37. Walker A, Pfitzner B, Neschen S, Kahle M, Harir M, Lucio M, Moritz F, Tziotis D, Witting M, Rothballer $\mathrm{M}$ et al: Distinct signatures of host-microbial meta-metabolome and gut microbiome in two C57BL/6 strains under high-fat diet. The ISME journal 2014, 8(12):2380-2396.

38. Chen S, Wu Q, Zhu L, Zong G, Li H, Zheng H, Zeng R, Lin X, Sun L: Plasma glycerophospholipid profile, erythrocyte n-3 PUFAs, and metabolic syndrome incidence: a prospective study in Chinese men and women. The American journal of clinical nutrition 2021, 114(1):143-153.

39. So SSY, Yeung CHC, Schooling CM, El-Nezami H: Targeting bile acid metabolism in obesity reduction: A systematic review and meta-analysis. Obesity reviews: an official journal of the International Association for the Study of Obesity 2020, 21(7):e13017.

40. Zhang Y, Bobe G, Revel JS, Rodrigues RR, Sharpton TJ, Fantacone ML, Raslan K, Miranda CL, Lowry MB, Blakemore PR et al: Improvements in Metabolic Syndrome by Xanthohumol Derivatives Are Linked to Altered Gut Microbiota and Bile Acid Metabolism. 2020, 64(1):e1900789. 
41. Yang Y, Chang Y, Wu Y, Liu H, Liu Q, Kang Z, Wu M, Yin H, Duan J: A homogeneous polysaccharide from Lycium barbarum: Structural characterizations, anti-obesity effects and impacts on gut microbiota. International journal of biological macromolecules 2021, 183:2074-2087.

\section{Appendix}

Appendices 1-3 are not available with this version.

\section{Figures}
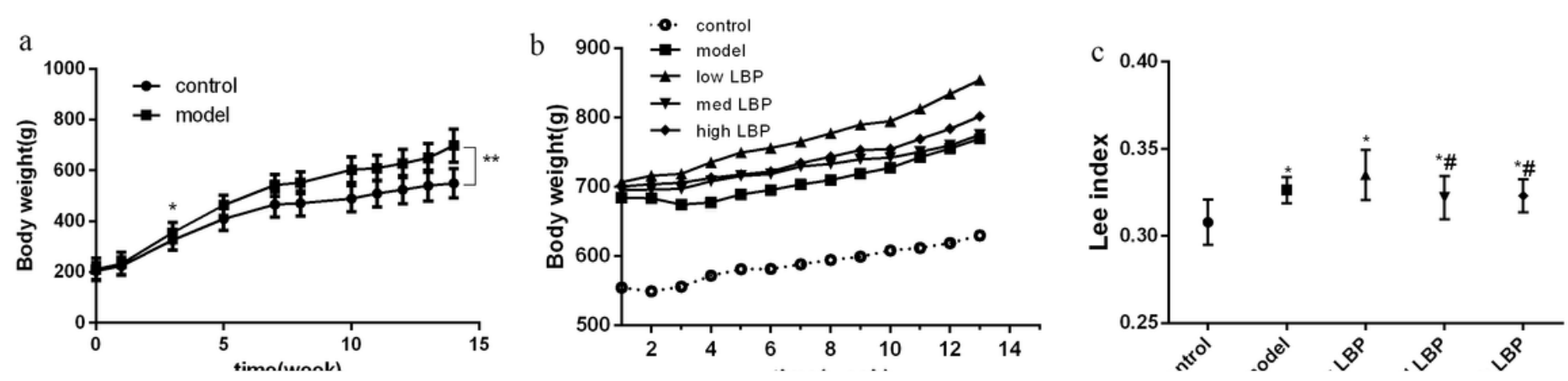

\section{Figure 1}

Determination of weight among all groups: a) Bodyweight during modeling; b) Bodyweight during intervention; c) Lee index. Lee index $=$ Bodyweight $(\mathrm{g}) 1 / 3 \times 1000 /$ body length $(\mathrm{cm})$ (control) control group; (model) model group; (low LBP) low dose group of LBP; (med LBP) medium-dose group of LBP; (high LBP) high dose group of LBP. Values are presented as mean $\pm S D, n=10$. *indicates the significant difference compared with control group $p<0.05$, \#indicates the significant difference intervention compared with model group $\mathrm{p}<0.05$. 

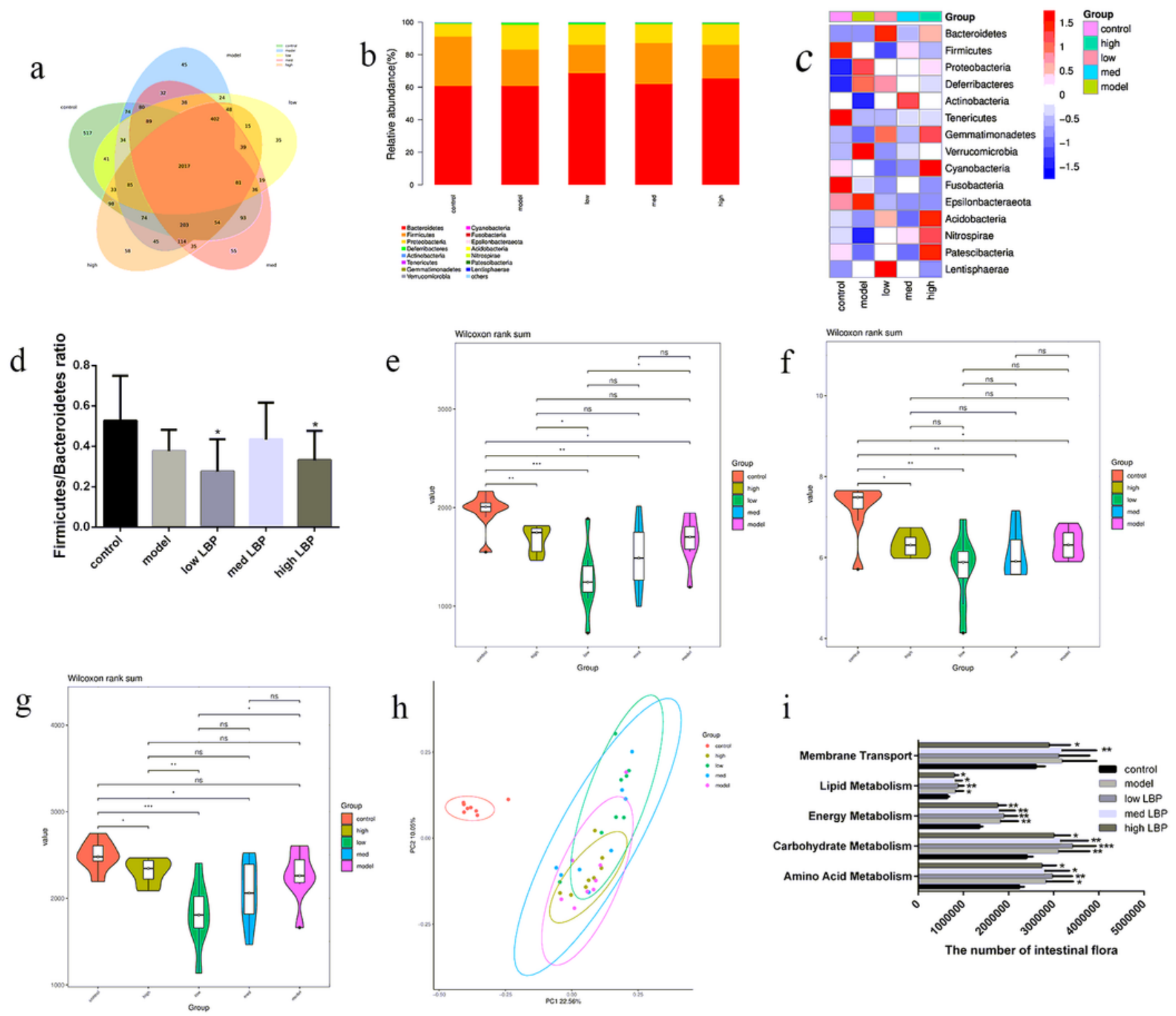

\section{Figure 2}

Effect of LBP on the compositions of gut microbiota. a) flower plot about the number of OTUs; $b$ ) comparison of relative abundance at phylum; c) heatmap of species among the five groups; $d$ ) the Firmicutes/Bacteroidetes ratio; $\alpha$-diversity analysis: e) observed species violin-plot, f) Shannon, and g) chao1 violin-plot; h) PCoA plot; i) Metabolic pathways from KEGG module predictions using 16S rRNA data with PICRUSt ( $n=8$ per group). Pairwise comparisons using Wilcoxon rank sum test; ${ }^{*} p<0.05$, $* * p<$ $0.01, \star \star \star p<0.001$ for versus model group; ns means the difference was not statistically significant. All the differences were analyzed using Kruskal-Wallis test; ${ }^{*} p<0.05 ;{ }^{\star \star} p<0.01$ for versus model group. 

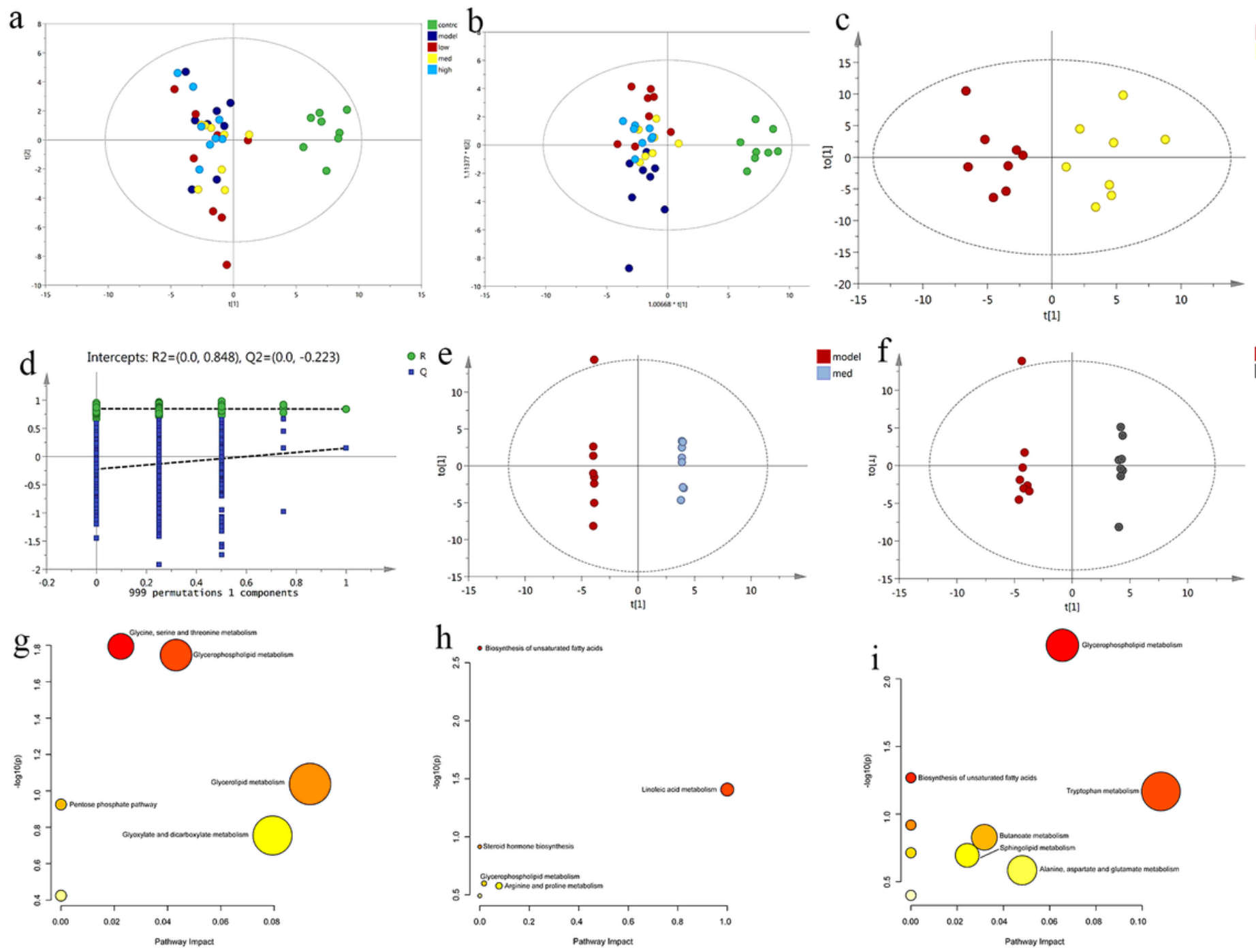

\section{Figure 3}

The results of metabolomics data analysis: a) unsupervised PCA score plots of metabolic for five groups; b) OPLS-DA score plots of metabolic for five groups; c) OPLS-DA score plots of metabolic between low LBP and model group; d) test robustness of OPLS-DA modeling compared low LBP to model; e) OPLS-DA score plots between med LBP and model group; f) OPLS-DA score plots between high LBP and model group; Metabolic pathway analysis result of differential metabolites $(\mathrm{p}<0.05$ of t-test after FDR adjusting) g) low LBP and model group; h) med LBP and model group; i) high LBP and model group. 
low LBP vs model med LBP vs model

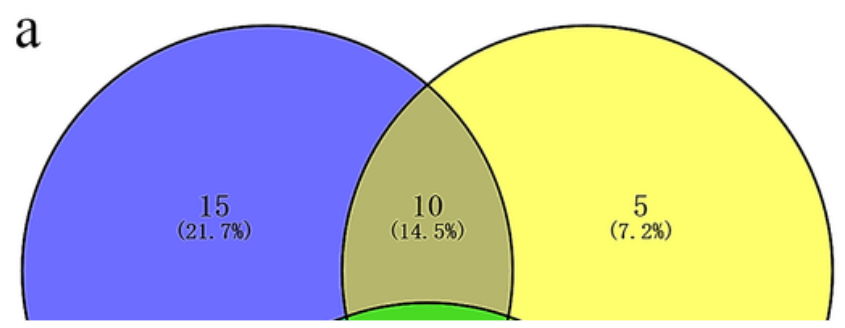

low LBP vs model

med LBP vs model

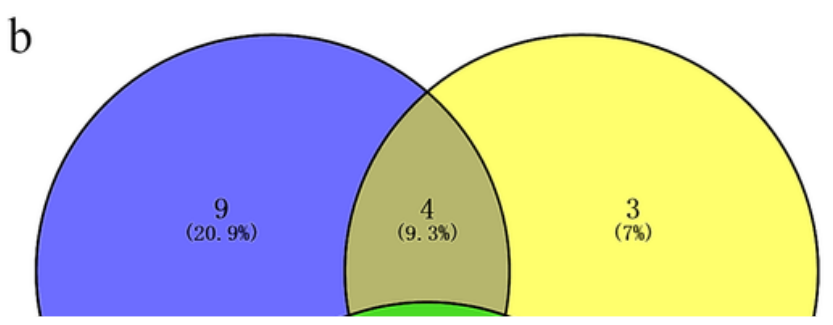

\section{Figure 4}

Venn diagram for differential metabolites. a) all different metabolites in compared groups; b) increased metabolites in compared groups. 

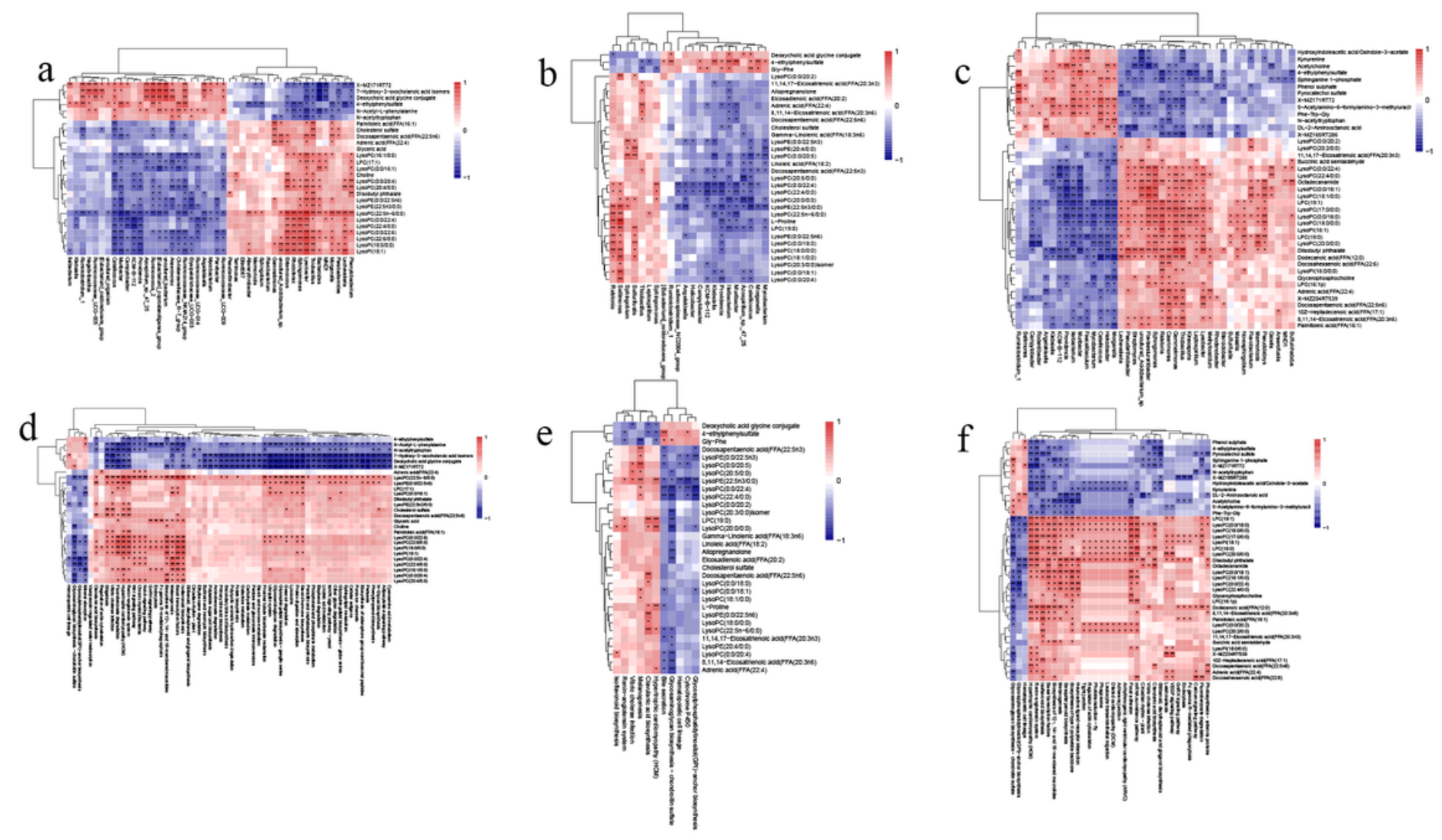

\section{Figure 5}

Different bacteria and metabolites correlation analysis and significant difference of heatmap figure. a) low LBP vs model; $b$ ) med LBP vs model; $c$ ) high LBP vs model. Different bacteria function predicted pathways and metabolites correlation analysis and significant difference of heatmap figure. d) low LBP vs model; e) med LBP vs model; f) high LBP vs model. Heat maps showed the positive (red) and negative (blue) correlation between the levels of genes in obese rats treated with different LBP. 